\title{
Effects of EBN on embryo implantation, plasma concentrations of reproductive hormones, and uterine expressions of genes of PCNA, steroids, growth factors and their receptors in rats
}

\begin{abstract}
This study was conducted to determine the effect of edible bird's nest (EBN) supplement on uterine function and embryo-implantation rate. A total of 24 adult female rats, divided equally into four groups, were treated with different doses of EBN for 8 weeks. In the last week of treatment, intact fertile male rats were introduced into each group (three per group) for overnight for mating. On day 7 post-mating (post-implantation), blood samples were collected from the hearts of anaesthetised rats that were later sacrificed. The uteri were removed for assessment of embryo implantation rate, histological and electron microscopic examination, and immunohistochemical analyses. Results showed that as the concentration of EBN supplemented increased, the pregnancy and embryo implantation rates were also increased in the treated groups; significantly at G3 and G4. Although histological evaluation did not show much difference among the groups, scanning electron microscopic examination showed enhanced development of elongated microvilli and pinopods in G4. Results also revealed upregulated expressions of epidermal growth factor (EGF), EGF receptor (EGFR), vascular endothelial growth factor (VEGF), proliferating cell nulear antigen (PCNA), and progesterone and estrogen receptors (P4R, E2R) in the uteri of treated groups. Moreover, plasma E2, P4, growth hormone $(\mathrm{GH})$ and prolactin $(\mathrm{P})$ levels were higher $(\mathrm{p}<0.05)$ in $\mathrm{G} 3$ and $\mathrm{G} 4$. The EBN increased the antioxidant (AO) and total AO capacities (TAC) and reduced oxidative stress (OS) levels in pregnant rats. In conclusion, findings of this study revealed that EBN enhances fertility and embryo implantation rate via promoting proliferation and differentiation of uterine structures as evidenced by the upregulation of the expressions of steroid receptors, EGF, EGFR, VEGF, and PCNA in the uterus. Furthermore, observations of improved growth of ultrastructural pinopods that assist in embryo attachment with uterine epithelium, increased concentrations of E2, P4, GH and P levels, as well as increased AO capacities with reduced OS levels in the treated groups might reflect additional possible mechanisms by which EBN enhances embryo implantation rate and pregnancy success.
\end{abstract}

Keyword: Antioxidants; EBN; Gravid uterus; Growth factors; Hormones; PCNA 